\title{
Media Use and the Cancer Communication Strategies of Cancer Survivors
}

\author{
Heesoo Yoon ${ }^{1}$, Minsung Sohn ${ }^{1}$, Minsoo Jung ${ }^{2}$ \\ ${ }^{1}$ Department of Public Health Science and BK21Plus Program in Public Health Science, Korea University, ${ }^{2}$ Department of Health Science, Dongduk \\ Women's University, Seoul, Korea
}

\begin{abstract}
Communication related to health not only substantially affects perceptions and behaviors related to health but is also positively associated with the extent of health-information seeking and the practice of preventive behavior. Despite the fact that the number of cancer survivors has increased dramatically, there are few studies of the lack of health information, factors which act as barriers, and the difficulties in follow-up care experienced by cancer survivors. Therefore, we reviewed media utilization and the types of media used by cancer survivors with regard to risk communication and suggested appropriate strategies for cancer communication. According to the results, health communication contributed to health promotion by providing health-related information, consolidating social support factors such as social solidarity and trust, and reducing anxiety. In particular, participatory health communication may establish preventive programs which reflect the needs of communities, expand accessibility to better quality healthcare, and intensify healthy living by reducing health inequalities. Therefore, when people do not have an intention to obtain cancer screening, we need to intervene to change their behavior, norms, and degrees of self-efficacy. The findings of this study may help those involved in building partnerships by assisting in their efforts to understand and communicate with the public.
\end{abstract}

(J Cancer Prev 2016;21:127-134)

Key Words: Cancer, Survivor, Health communication, Self efficacy

\section{INTRODUCTION}

Health communication is a process by which understandable health-related information is scientifically developed and strategically disseminated so that individuals and communities can easily access it in numerous ways, such as from mass communication, journalism and marketing sources. ${ }^{1}$ Specifically, during a health campaign, because community members are not passive consumers but partners who participate in the framing of a message, it is important to recognize their health perceptions and needs. ${ }^{1,2}$ Furthermore, during the process of developing heath communication messages, it is necessary to facilitate health-information seeking behaviors (HISBs) while also considering the determinants (e.g., race, education, income, and lifestyles) in a health and social context. ${ }^{3.4}$ However, the great amount of effort expended to improve HISBs is often overlooked in South Korea. In particular, after 2000, despite the fact that the number of cancer survivors has increased dramatically, there are few studies of the lack of health information, factors which act as barriers, and the difficulties in follow-up care experienced by cancer survivors. ${ }^{5.6}$

Today, communication related to health not only substantially affects perceptions and behaviors related to health but is also positively associated with the extent of health-information seeking and the practice of preventive behavior. ${ }^{7,8}$ In detail, conventional mass media channels, including television, newspaper and radio, are beneficial for expanding perception through the initial diffusion of health information, while individual

Received August 27, 2016, Accepted September 4, 2016

Correspondence to: Minsoo Jung

Department of Health Science, Dongduk Women's University, 60 Hwarang-ro 13-gil, Seongbuk-gu, Seoul 02748, Korea

Tel: +82-2-940-4483, Fax: +82-2-940-4480, E-mail: mj748@dongduk.ac.kr, ORCID: Minsoo Jung, http://orcid.org/0000-0003-3317-6507

Copyright (C) 2016 Korean Society of Cancer Prevention

(c) This is an Open Access article distributed under the terms of the Creative Commons Attribution Non-Commercial License (http://creativecommons.org/licenses/by-nc/4.0) which permits unrestricted non-commercial use, distribution, and reproduction in any medium, provided the original work is properly cited. 
channels, including family, friends and colleagues, are advantageous for those who seek to change their health-related behaviors. ${ }^{9,10}$ Moreover, participatory health communication can establish preventive programs which reflect the needs of communities, expand accessibility to better quality healthcare, and intensify healthy living by reducing health inequalities. ${ }^{11}$ Therefore, this study investigated media utilization and the types of media used by cancer survivors with regard to risk communication and suggested appropriate strategies for cancer communication. The findings can help those involved in building partnerships by assisting in their efforts to understand and communicate with the public. They can also help socially underprivileged people to increase their health aptitudes. ${ }^{12}$

\section{CANCER AND RISK COMMUNICATION}

Risk communication refers to a purposeful exchange of information between stakeholders regarding health-related and environmental risks. ${ }^{13}$ In modern society, mass media is a pivotal channel of communication in which a variety of risks are introduced as an agenda. Because fear in a society can affect people's perceptions and behaviors, people are more likely to feel anxious, fearful and nervous when they are exposed by media to information about risky circumstances. ${ }^{14}$ As people usually tend to speculate on risks not from their experiences but from the content reported by the media, they are more susceptible to unfamiliar risks. ${ }^{15}$ As a case in point, environmental reporting not only has a great influence on citizens' behaviors, perceptions and on the formation of public opinion, but it also changes perceptions according to media framing. ${ }^{16,17}$ Likewise, media plays an important role in transmitting crisis circumstances not experienced by the consumer, functioning to provide significant criteria related to risk management and for assessing the ability to cope. ${ }^{14}$

In order to deliver information about the risks of chronic diseases such as cancer effectively through current forms of media, accuracy in reporting is paramount. Today, as media is deputed to collect, publish, and disseminate information, and the degree to which media reports are closer to actual events is very significant; that is, the accuracy of an article is one of the most pivotal factors. Indeed, accuracy is a rudimentary value in journalism. ${ }^{18}$ Furthermore, given that the contexts of the reported contents as well as the interpretations, discussions and publicly communicated messages are feasibly based on facts, if there are problematic facts, other related facts will also be flawed. ${ }^{18}$ However, it is implausible that media reporting is accurate when reporting news related to cancer. For example, carcinogens, referring to substances that cause cancer or increase the occurrence of cancer, require active communication so as not to cause fear and impede prevention, screening and treatments. ${ }^{19}$ However, because diseases such as cancer can have a negative effect on the behaviors of the public due to their lack of experience or uncertainties about the nature of cancer, the media and how cancer is reported play an important role. ${ }^{20,21}$

In order to judge accuracy in reporting, four standards are required. First, it is necessary to represent carcinogenic levels properly. This refers to the degree of carcinogenic risk as designated by the International Agency for Research on Cancer (IARC), an organization affiliated with the World Health Organization. Researchers at international cancer research centers and other related experts evaluate the carcinogenicity of candidate carcinogenic substances through conferences. ${ }^{22}$ To verify sufficient epidemiological data about the human body, substances which are carcinogenic to humans are categorized into what is termed group 1 carcinogens. On the other hand, despite the existence of limited epidemiological data, if there is sufficient data derived from animal studies, probable substances carcinogenic to humans are classified as group 2A carcinogens. In addition, substances possibly carcinogenic to humans are categorized into group 2B in the event of insufficient data from both epidemiological studies and animal studies. Substances not classifiable as carcinogenic to humans and most likely not carcinogenic to humans are classified into group 3 and group 4 , respectively. Regarding the second standard, it is necessary to use the official names of carcinogens assigned by the IARC. For instance, while dichloromethane is an official name assigned by the IARC, dichloroethene is neither an official name nor is it assigned as a carcinogen. Hence, though the name is similar, because carcinogenicity is different, the exact use of the carcinogen name is cardinal for properly transmitting information. Third, it is necessary to represent the limitations of carcinogenic exposure. People are not rational creatures that do not judge rationally and logically but are cognitive misers that do not make the psychological effort and judge rapidly, therefore using heuristic mechanisms to find answers quickly rather than finding accurate ones slowly. ${ }^{23}$ Tversky and Kahneman ${ }^{24}$ suggested anchoring, which is one of the heuristics considered as a mechanism behind judgments. When judging values, anchoring becomes a standard and influences decision making. Moreover, when reporting carcinogens, anchoring acts as key standard point. ${ }^{25}$ The standard of a carcinogen refers to carcinogenic levels which barely affect humans when they are exposed to it or ingest it. If a news viewer receives information concerning standards in a carcinogen-related 
article, it is beneficial for them to perceive the risk. Lastly, reporting requirements should be covered and written as an article by professional reporters.

\section{MEDIA USE AND COMMUNICATION INEQUALITY IN CANCER SURVIVORS}

For cancer, one of the major chronic diseases, postoperative management and follow-up care are as important as cancer prevention. For optimal management, patients as well as health providers should make an effort to attain follow-up care, which is why cancer communication is considered as a significant factor to improve the odds of surviving cancer. ${ }^{3,4,26}$ In terms of cancer communication, we can easily find a vulnerable population. For example, although the five-year relative survival rates of children and adolescents with cancer have increased to more than $80 \%$ in the USA, three out of four still have chronic diseases, and half of them are of the life-threatening types. ${ }^{27-29}$ Due to development of cancer treatments, if treatment starts earlier, the morbidity and mortality rates can be decreased. However, without proper follow-up care, cancer survivors can suffer a fatal prognosis. ${ }^{30}$ According to the clinical practice guidelines of the Children's Oncology Group established by the Institute of Medicine, adolescent cancer survivors should quit smoking, eat a healthy diet, and take part in physical activities to decrease co-morbidity rates. $^{31}$ However, in spite of these recommendations, childhood-adolescent cancer survivors are more likely to engage in risky behaviors than healthy people. ${ }^{32,33}$ In fact, it was reported that one third of childhood-adolescent cancer survivors smoke. ${ }^{34,35}$ Additionally, more than half of cancer survivors do not conform to recommended diet and physical activity guidelines. $^{36,37}$ Despite public health interventions in the USA $^{38,39}$ there are few population-based strategies which can be established through cancer communication.

Recently, health information in the media has dramatically increased, and there is increasingly sufficient evidence that active information seeking and routine exposure to health information have a great impact on the health knowledge, confidence and behaviors of both ordinary populations and patients. ${ }^{26,40}$ People who seek health information via the media have better cancer-related knowledge, eat more vegetables and fruit, exercise more, and undergo more cancer screening procedures such as colonoscopies and mammograms compared to those who do not seek such information. ${ }^{41-43}$ Cancer survivors who actively seek follow-up treatment show better prognoses than those who do not. ${ }^{3,44,45}$ However, not all people equally benefit from the media exposure effect, and this is regarded as a form of communication inequality. ${ }^{46}$ People who have lower incomes are not only less likely to be exposed to cancer information but also have more difficulty in handling it as well. ${ }^{3,19,41,44}$ Furthermore, cancer survivors of a low socioeconomic status (SES) are more likely to seek information about loans or employment than they are about health. ${ }^{47}$

In spite of the significance of cancer communication, it has been criticized in that the inequality gap is not being reduced. ${ }^{48-50}$ For childhood-adolescent cancer survivors, although there are information sources such as websites, the quality of the information was poor, and the ability of these websites to supply the necessary information was restricted. ${ }^{51,52}$ There are not a few studies about utilizing information by cancer survivors. For example, Knijnenburg et al. ${ }^{53}$ reported that $49 \%$ of cancer survivors sought health information through the Internet, yet it was difficult hard to find information about the search contents, and the sample size was also small in their study. Therefore, it is necessary to investigate media use as it pertains to health and to suggest proper strategies for cancer communication. In doing this, informative media can be developed and the types of cancer survivors and their characteristics can be revealed. ${ }^{5457}$

Nagler et al. $^{58}$ analyzed data derived from Partnership for Health-2, a project conducted through a web-based campaign for nonsmoking among childhood-adolescent cancer survivors. According to their results, smokers who were childhood-adolescent cancer survivors rarely seek health-related information on the Internet. Their rate of using health-related media is considerably low compared to that of adult cancer survivors. For example, only one third of them seek information, and they do so less than once a month. ${ }^{3,44,45}$ Consequently, given that data pertaining to the use of health-related media is not abundant, ${ }^{48,49,53}$ more relevant factors such as online information seeking or exposure to media should be included in a comprehensive survey about cancer survivors.

We should pay more attention to the following cases. First, cancer survivors with relatively low education levels are less likely to seek health-related information online compared to those with high education levels. ${ }^{3,41,44}$ As it is, cancer survivors who can easily access the internet show similar trends. Second, communication inequalities can aggravate health disparities, as the use of media sources varies depending on the subgroup of cancer survivors (Fig. 1). ${ }^{46,53,59,60}$ Thus, cancer communication strategies should be specifically established based on the characteristics of vulnerable groups subdivided from larger groups of cancer survivors. For instance, when cancer survivors 


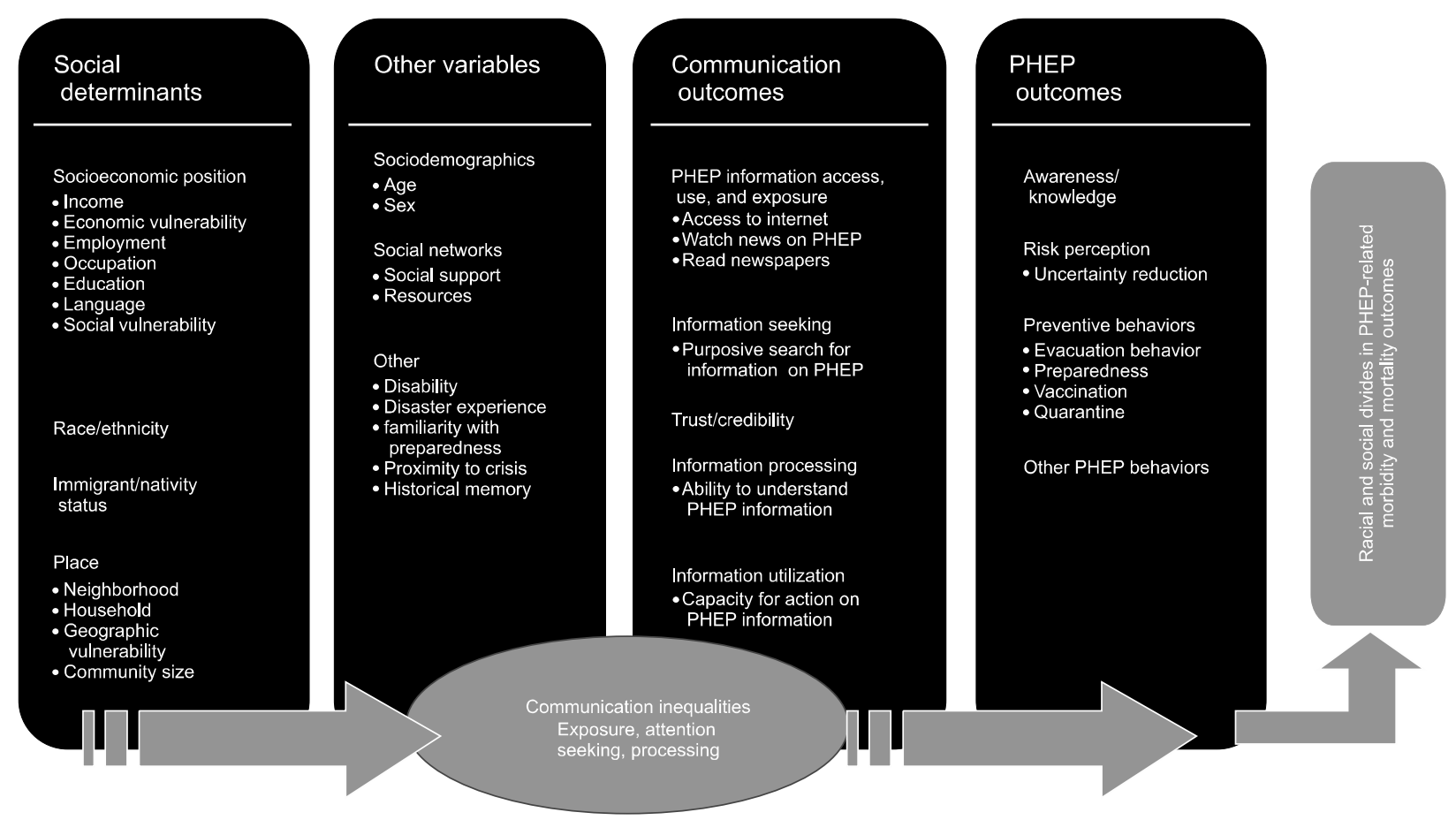

Figure 1. Structural influence model of communication inequalities. Data from Jung et al. ${ }^{60}$ (Vaccine 2013:31:4860-6) with original copyright holder's permission. PHEP, public health emergency preparedness.

who are eager to quit smoking seek information or support, they tend to rely on a certain type of media, such as online sources rather than printed media or broadcasting. ${ }^{58}$ Thus, to promote healthy behaviors and reduce risky behaviors, it is necessary to develop evidence-based programs and integrate them into treatment services for cancer survivors, thus triggering them to engage in cancer communication themselves..$^{55.61}$ Additionally, clinicians should recommend healthy behaviors as follow-up care. ${ }^{37}$ That is, by understanding the means by which cancer survivors obtain health-related information and by understanding how they consume it, clinicians can support cancer survivors to obtain health-related information from more reliable information sources. For a group that stops or avoids searching for information, their characteristics must be studied and specially managed. ${ }^{4}$

Face-to-face talks with cancer survivors can represent a crucial opportunity in that they inspire patients to engage in better health behaviors and they help patients gain more reliable information. This support is especially important to population with low education levels, who have had difficulty in dealing with healthcare issues. Due to the fact that it is difficult for them to obtain health-related information, they also have limited opportunities for follow-up care. ${ }^{62}$ As a result, cancer communication based on use of health-related media by cancer survivors can contribute to reducing potential communication inequalities, as cancer survivors can obtain information from high-quality information sources and thus meet their needs.

\section{DEVELOPING STRATEGIES FOR CANCER COMMUNICATION}

Population-based health promotion begins with health communication to improve social conditions and to provide information that helps people practice healthy behaviors. ${ }^{63}$ According to this point, it is necessary to recognize in targeted campaign groups the ability to communicate and their social support and self-efficacy and to investigate barriers to healthy behaviors such as preventive health screening. ${ }^{64}$ The more social support is improved, the more treatments of diseases and self-efficacy for cancer communication will increase. The term self-efficacy refers to a belief that the public can control their motives, behaviors and social environments. ${ }^{63,65}$

Cancer, one of the main causes of death, is characterized by uncertainty, bringing a desire to understand new characteristics of cancer and communicate them. ${ }^{7}$ Health status is usually affected by not only individuals' habits, including how much they smoke, drink, exercise, and their stress levels, but also by social structure and cultural factors such as age, gender, class, religion, 
race, and ethnicity. Because a campaign's message should be based on an understanding of the subtle differences in the context of social structure and culture, it is necessary to take a strategic approach to examine health issues and efforts to improve health promotion which are focused on specific a culture and context. ${ }^{66}$ That is, it is necessary to select the proper language, the strategy of the message, and to decide on the channel while considering the cultural characteristics, needs and health literacy of the public. ${ }^{67,68}$

In terms of contextual construction, health is a social composition and a process of incidence and treatment of a disease and is affected by aspects of social networks such as the social context, family and friends. ${ }^{66}$ Social support involves contact from family, friends, and colleagues to obtain advice about health. Unofficial sources are included, as they can provide physical care and psychological support. Social support can reduce the uncertainty surrounding the situation and increase disease controllability levels. ${ }^{8}$ For example, when making decisions about treatment, breast cancer patients who have a spouse are more active and cooperative and more satisfied with the treatment. ${ }^{3}$ Furthermore, if they receive emotional and informational support from their spouse, family and friends, they increase their levels of participation and satisfaction with regard to their treatment. ${ }^{69}$ As a result, communication through various interpersonal and media channels provides social support, strengthens self-efficacy, and increases the possibility of practicing healthy behaviors.

There is sufficient evidence that social support greatly impacts health status and that online social support also affects one's health. ${ }^{5,63,64,70}$ As the perception that screening is unnecessary or expensive and shame associated with the screening process can be barriers to screening, it is necessary to strengthen social support through communication. ${ }^{66}$ Specifically, Japanese-American or Vietnamese-American women rely more on their family when they need health-related information and help, which shows the importance of social support. In the case of Hong Kong, a lack of perception of health, a low education level, and insufficient health-related information were obstacles to proper cervical cancer screening. ${ }^{71}$

Self-efficacy is a significant factor when attempting to change health-related behaviors as it relates to a belief that successful cancer screening can prevent cancer. ${ }^{66}$ When people have strong self-efficacy, they are more likely to be attentive to health messages and to practice healthy behavior. ${ }^{72-74}$ With strong self-efficacy, people become good at controlling emotions and limiting risky health behaviors. ${ }^{69}$ Regarding the breast self-examination rate among Mexican-American women, high self-efficacy is associated with preventive behaviors such as eating a balanced diet, exercise and not smoking. ${ }^{73}$ However, in the event of lower self-efficacy, such patients avoid cancer screening and feel nervous about it. ${ }^{64}$

Culturally appealing to insensitive message stresses an individual's responsibilities and rights, and this is related to a tendency to ignore factors such as cultural beliefs about prognosis and treatment and the rudeness of medical providers. ${ }^{66}$ In a cancer prevention campaign performed by National Cancer Plan UK in 2007, the findings revealed that a group with low SES had a high cancer mortality rate. ${ }^{75}$ Those who earn low incomes, non-whites and sexual minorities are often alienated from health communication, and health-related information is not aimed at such people. ${ }^{66,76}$ Factors such as race, education level, regularly meeting doctors, familiar cancer patients, and exposure to public advertisements are also associated with cancer screening behavior. $^{73,77}$ These cultural factors had an influence on the low rate of breast cancer screening among Asian-American women as well. ${ }^{78}$ With regard to living in the USA for a short time, with poor English and modesty, minorities experience difficulty in communicating about their health and diseases. ${ }^{78}$

\section{CONCLUSION}

Health communication contributes to health promotion by providing health-related information, consolidating social support factors such as social solidarity and trust, and reducing anxiety. ${ }^{8}$ People with improved social support become more attentive to messages regarding health and adhere to preventive health behaviors. ${ }^{72}$ Public health campaigns are a form of communication that enables people to solve the problems of discrimination and stigmas about health issues, thus contributing to health promotion for minorities or vulnerable groups. ${ }^{46}$ The aims of intervention campaigns should vary depending on the target group. For instance, when people intend to obtain cancer screening but do not in fact follow through on this, it is necessary to focus on removing environmental barriers and developing communication strategies. However, when people do not have an intention to obtain cancer screening, we should intervene to change their behavior, norms and degrees of self-efficacy.

Greater levels of social support, self-efficacy and health literacy, which increase the intention to engage in cancer communication, lead to more healthy behavior. Therefore, this shows that promoting empowerment, which strengthens various forms of social support and self-controllability by the public, is needed. It is a barrier to cancer screening and treatment for black men in the USA when they lack cancer-related knowledge, have a fear of 
cancer, feel disgust about cancer screening, distrust the healthcare system, and avoid cancer communication. ${ }^{79}$ Cancer communication through face-to-face meetings can abate uncertainty about cancer, facilitate controllability, and can be beneficial for spreading messages about cancer prevention. ${ }^{8}$ As a result, messages about health reinforce self-efficacy and thus encourage patients to fight cancer while also providing support. ${ }^{64}$

Participatory health campaigns develop partnerships not with experts but via interactions among patient, community members, health service providers and participation by the patient. Campaigns based on speaking from above often systematically ignore voices from the surroundings; however, campaigns based on speaking from below reflect voices from the surroundings when it comes to defining a problem, organizing a solution, and formulating a policy. ${ }^{80}$ Cancer communication can succeed in bottom-up campaigns based on social networks and with support of cancer survivors. ${ }^{67,73}$ In that optimal care can be achieved by medical effectiveness, communication, and human outreach, it is necessary to stir up and consolidate cancer communication strategies.

\section{ACKNOWLEDGMENTS}

This work was supported by the National Research Foundation of Korea Grant funded by the Korean Government (NRF-2015R1C1A1A01051608).

\section{CONFLICTS OF INTEREST}

No potential conflicts of interest were disclosed.

\section{REFERENCES}

1. Bernhardt JM. Communication at the core of effective public health. Am J Public Health 2004:94:2051-3.

2. Southwell BG. Audience construction and AIDS education efforts: Exploring communication assumptions of public health interventions. Critic Public Health 2000;10:313-9.

3. Jung M, Ramanadhan S, Viswanath K. Effect of information seeking and avoidance behavior on self-rated health status among cancer survivors. Patient Educ Couns 2013;92:100-6.

4. McCloud RF, Jung M, Gray SW, Viswanath K. Class, race and ethnicity and information avoidance among cancer survivors. $\mathrm{Br} \mathrm{J}$ Cancer 2013;108:1949-56.

5. Amankwah E, Ngwakongnwi E, Quan H. Why many visible minority women in Canada do not participate in cervical cancer screening. Ethn Health 2009;14:337-49.

6. Jung M. Cancer control and the communication innovation in South Korea: implications for cancer disparities. Asian Pac J Cancer Prev 2013;14:3411-7.
7. Niederdeppe J, Frosch DL, Hornik RC. Cancer news coverage and information seeking. J Health Commun 2008;13:181-99.

8. Stephens KK, Rimal RN, Flora JA. Expanding the reach of health campaigns: community organizations as meta-channels for the dissemination of health information. J Health Commun 2004;9 Suppl 1:97-111.

9. Haider M, Kreps GL. Forty years of diffusion of innovations: utility and value in public health. J Health Commun 2004:9 Suppl 1:3-11.

10. Yanovitzky I, Blitz CL. Effect of media coverage and physician advice on utilization of breast cancer screening by women 40 years and older. J Health Commun 2000;5:117-34.

11. Thomas SB, Fine MJ, Ibrahim SA. Health disparities: the importance of culture and health communication. Am J Public Health 2004:94:2050.

12. Dutta MJ. Communicating about culture and health: theorizing culture-centered and cultural sensitivity approaches. Commun Theory 2007; 17:304-28.

13. Covello V, von Winterfeldt D, Slovic P. Risk communication: a review of the literature. Risk Abstract 1986;3:171-82.

14. Sherrill D, Viegi G. On modeling longitudinal pulmonary function data. Am J Respir Crit Care Med 1996;154:S217-22.

15. Allan S. Media, risk and science. Buckingham, Open University Press, 2002.

16. Cohen J. Defining identification: a theoretical look at the identification of audiences with media characters. Mass Commun Soc 2001:4:245-64.

17. Jensen JD, Hurley RJ. Third-person effects and environment: social distance, social desirability, and presumed behavior. J Commun 2005:55:42-56.

18. Kovach B, Rosenstiel T. The elements of journalism: what newspeople should know and the public should expect. New York, Crown Publishers, 2007.

19. Viswanath K, Breen N, Meissner H, Moser RP, Hesse B, Steele WR, et al. Cancer knowledge and disparities in the information age. J Health Commun 2006;11 Suppl 1:1-17.

20. Talosig-Garcia M, Davis SW. Information-seeking behavior of minority breast cancer patients: an exploratory study. J Health Commun 2005; 10 Suppl 1:53-64.

21. Whiteman MK, Cui Y, Flaws JA, Langenberg P, Bush TL. Media coverage of women's health issues: is there a bias in the reporting of an association between hormone replacement therapy and breast cancer? J Womens Health Gend Based Med 2001;10:571-7.

22. International Agency for Research on Cancer. IARC monographs on the evaluation of carcinogenic risks to humans. 2008. http:// monographs.iarc.fr/ENG/Monographs/PDFs/index.php. Accessed May 20, 2016.

23. Taylor SE. A categorization approach to stereotyping. In: Hamilton DL, ed. Cognitive Process in Stereotyping and Intergroup Behavior. Hillsdale, NJ, Erlbaum Associates, pp 83-114, 1981.

24. Tversky A, Kahneman D. Judgment under uncertainty: heuristics and biases. Science 1974;185:1124-31.

25. Payne JW, Bettman JR, Johnson E. Behavioral decision research: a constructive processing perspective. Ann Rev Psychol 1992;43: 87-131.

26. Viswanath K, Nagler RH, Bigman-Galimore CA, McCauley MP, Jung $\mathrm{M}$, Ramanadhan $\mathrm{S}$. The communications revolution and health inequalities in the 21st century: implications for cancer control. Cancer Epidemiol Biomarkers Prev 2012;21:1701-8. 
27. American Cancer Society. Cancer facts \& figures 2012. Atlanta, GA, American Cancer Society, 2012.

28. Geenen MM, Cardous-Ubbink MC, Kremer LC, van den Bos C, van der Pal HJ, Heinen RC, et al. Medical assessment of adverse health outcomes in long-term survivors of childhood cancer. JAMA 2007:297:2705-15.

29. Oeffinger KC, Mertens AC, Sklar CA, Kawashima T, Hudson MM, Meadows AT, et al. Chronic health conditions in adult survivors of childhood cancer. N Engl J Med 2006;355:1572-82.

30. Hewitt ME, Weiner SL, Simone JV. Childhood cancer survivorship: improving care and quality of life. Washington, DC, National Academies Press, 2003.

31. Landier W, Bhatia S, Eshelman DA, Forte KJ, Sweeney T, Hester $\mathrm{AL}$, et al. Development of risk-based guidelines for pediatric cancer survivors: the Children's Oncology Group Long-Term Follow-Up Guidelines from the Children's Oncology Group Late Effects Committee and Nursing Discipline. J Clin Oncol 2004:22:4979-90.

32. Klosky JL, Howell CR, Li Z, Foster RH, Mertens AC, Robison LL, et al. Risky health behavior among adolescents in the childhood cancer survivor study cohort. J Pediatr Psychol 2012;37:634-46.

33. Phillips-Salimi CR, Lommel K, Andrykowski MA. Physical and mental health status and health behaviors of childhood cancer survivors: findings from the 2009 BRFSS survey. Pediatr Blood Cancer 2012;58:964-70.

34. Emmons K, Li FP, Whitton J, Mertens AC, Hutchinson R, Diller L, et al. Predictors of smoking initiation and cessation among childhood cancer survivors: a report from the childhood cancer survivor study. J Clin Oncol 2002;20:1608-16.

35. Haupt R, Byrne J, Connelly RR, Mostow EN, Austin DF, Holmes GR, et al. Smoking habits in survivors of childhood and adolescent cancer. Med Pediatr Oncol 1992;20:301-6.

36. Badr H, Paxton RJ, Ater JL, Urbauer D, Demark-Wahnefried W. Health behaviors and weight status of childhood cancer survivors and their parents: similarities and opportunities for joint interventions. J Am Diet Assoc 2011;111:1917-23.

37. Stolley MR, Restrepo J, Sharp LK. Diet and physical activity in childhood cancer survivors: a review of the literature. Ann Behav Med 2010;39:232-49.

38. Emmons KM, Puleo E, Mertens A, Gritz ER, Diller L, et al. Long-term smoking cessation outcomes among childhood cancer survivors in the partnership for health study. J Clin Oncol 2009:27:52-60.

39. Blaauwbroek R, Bouma MJ, Tuinier W, Groenier KH, de Greef MH, Meyboom-de Jong B, et al. The effect of exercise counselling with feedback from a pedometer on fatigue in adult survivors of childhood cancer: a pilot study. Support Care Cancer 2009;17:1041-8.

40. Viswanath K. Science and society: the communications revolution and cancer control. Nat Rev Cancer 2005:5:828-35.

41. Kelly B, Hornik R, Romantan A, Schwartz JS, Armstrong K, DeMichele A, et al. Cancer information scanning and seeking in the general population. J Health Commun 2010;15:734-53.

42. Lewis N, Martinez LS, Freres DR, Schwartz JS, Armstrong K, Gray SW, et al. Seeking cancer-related information from media and family/friends increases fruit and vegetable consumption among cancer patients. Health Commun 2012;27:380-8.

43. Shim M, Kelly B, Hornik R. Cancer information scanning and seeking behavior is associated with knowledge, lifestyle choices, and screening. J Health Commun 2006;11 Suppl 1:157-72.

44. Nagler RH, Gray SW, Romantan A, Kelly BJ, DeMichele A,
Armstrong $\mathrm{K}$, et al. Differences in information seeking among breast, prostate, and colorectal cancer patients: results from a population-based survey. Patient Educ Couns 2010;81 Suppl:S54-62.

45. Mayer DK, Terrin NC, Kreps GL, Menon U, McCance K, Parsons SK, et al. Cancer survivors information seeking behaviors: a comparison of survivors who do and do not seek information about cancer. Patient Educ Couns 2007:65:342-50.

46. Viswanath K. Public communications and its role in reducing and eliminating health disparities. In: Thomson GE, Mitchell F, Williams MB, eds. Examining the Health Disparities Research Plan of the National Institutes of Health: Unfinished Business. Washington, DC, National Academies Press, pp 215-53, 2006.

47. Galarce EM, Ramanadhan S, Weeks J, Schneider EC, Gray SW, Viswanath K. Class, race, ethnicity and information needs in post-treatment cancer patients. Patient Educ Couns 2011;85:432-9.

48. Dyson GJ, Thompson K, Palmer S, Thomas DM, Schofield P. The relationship between unmet needs and distress amongst young people with cancer. Support Care Cancer 2012;20:75-85.

49. Keegan TH, Lichtensztajn DY, Kato I, Kent EE, Wu XC, West MM, et al. Unmet adolescent and young adult cancer survivors information and service needs: a population-based cancer registry study. J Cancer Surviv 2012;6:239-50.

50. Zebrack B. Information and service needs for young adult cancer survivors. Support Care Cancer 2009;17:349-57.

51. Stinson JN, White M, Breakey V, Chong AL, Mak I, Low KK, et al. Perspectives on quality and content of information on the internet for adolescents with cancer. Pediatr Blood Cancer 2011;57: 97-104.

52. Freyer DR, Mattano LJ. Information and resources for young adults and adolescents with cancer. In: Bleyer WA, Barr RD, eds. Cancer in Adolescents and Young Adults. New York, Springer Verlag, pp 469-86, 2007.

53. Knijnenburg SL, Kremer LC, van den Bos C, Braam KI, Jaspers MW. Health information needs of childhood cancer survivors and their family. Pediatr Blood Cancer 2010;54:123-7.

54. de Moor JS, Puleo E, Ford JS, Greenberg M, Hodgson DC, Tyc VL, et al. Disseminating a smoking cessation intervention to childhood and young adult cancer survivors: baseline characteristics and study design of the partnership for health-2 study. BMC Cancer 2011;11:165.

55. Emmons KM, Puleo E, Sprunck-Harrild K, Ford J, Ostroff JS, Hodgson D, et al. Partnership for health-2, a web-based versus print smoking cessation intervention for childhood and young adult cancer survivors: randomized comparative effectiveness study. J Med Internet Res 2013;15:e218.

56. Knijnenburg SL, Kremer LC, Versluys AB, Braam KI, Mud MS, van der Pal HJ, et al. Evaluation of a patient information website for childhood cancer survivors. Support Care Cancer 2013;21:919-26.

57. Oeffinger KC, Hudson MM, Mertens AC, Smith SM, Mitby PA, Eshelman-Kent DA, et al. Increasing rates of breast cancer and cardiac surveillance among high-risk survivors of childhood Hodgkin lymphoma following a mailed, one-page survivorship care plan. Pediatr Blood Cancer 2011;56:818-24.

58. Nagler RH, Puleo E, Sprunck-Harrild K, Viswanath K, Emmons $\mathrm{KM}$. Health media use among childhood and young adult cancer survivors who smoke. Support Care Cancer 2014:22:2497-507.

59. Nagler RH, Puleo E, Sprunck-Harrild K, Emmons KM. Internet use among childhood and young adult cancer survivors who smoke: implications for cessation interventions. Cancer Causes Control 
2012;23:647-52

60. Jung M, Lin L, Viswanath K. Associations between health communication behaviors, neighborhood social capital, vaccine knowledge, and parents' H1N1 vaccination of their children. Vaccine 2013;31:4860-6.

61. Campo RA, Rowland JH, Irwin ML, Nathan PC, Gritz ER, Kinney AY. Cancer prevention after cancer: changing the paradigm: a report from the American Society of Preventive Oncology. Cancer Epidemiol Biomarkers Prev 2011:20:2317-24.

62. Barakat LP, Schwartz LA, Szabo MM, Hussey HM, Bunin GR. Factors that contribute to post-treatment follow-up care for survivors of childhood cancer. J Cancer Surviv 2012;6:155-62.

63. Beaudoin CE, Thorson E, Hong T. Promoting youth health by social empowerment: a media campaign targeting social capital. Health Commun 2006;19:175-82.

64. Hubbell AP. Mexican American women in a rural area and barriers to their ability to enact protective behaviors against breast cancer. Health Commun 2006;20:35-44.

65. Campo S, Mastin T. Placing the burden on the individual: overweight and obesity in African American and mainstream women's magazines. Health Commun 2007;22:229-40.

66. Kline KN. Cultural sensitivity and health promotion: assessing breast cancer education pamphlets designed for African American women. Health Commun 2007:21:85-96.

67. Patel K, Bhaskaran M, Dani D, Reddy K, Singhal PC. Role of heme oxygenase-1 in morphine-modulated apoptosis and migration of macrophages. J Infect Dis 2003;187:47-54.

68. Davis TC, Gazmararian J, Kennen EM. Approaches to improving health literacy: lessons from the field. J Health Commun 2006;11:551-4.

69. Han WT, Collie K, Koopman C, Azarow J, Classen C, Morrow GR, et al. Breast cancer and problems with medical interactions: relationships with traumatic stress, emotional self-efficacy, and so- cial support. Psychooncology 2005;14:318-30.

70. Freimuth VS, Quinn SC. The contributions of health communication to eliminating health disparities. Am J Public Health 2004; 94:2053-5.

71. Abdullah AS, Leung TY. Factors associated with the use of breast and cervical cancer screening services among Chinese women in Hong Kong. Public Health 2001;115:212-7.

72. Andsager JL, Powers A. Framing women's health with a sense-making approach: magazine coverage of breast cancer and implants. Health Commun 2001;13:163-85.

73. Egbert N, Parrott R. Self-efficacy and rural women's performance of breast and cervical cancer detection practices. J Health Commun 2001:6:219-33.

74. Porto MP. Fighting AIDS among adolescent women: effects of a public communication campaign in Brazil. J Health Commun 2007;12:121-32.

75. Daniel R, Simpson M. The development of a local cancer awareness communications campaign. J Commun Healthc 2008;1:408-21.

76. Aldoory L. Making health communications meaningful for women: factors that influence involvement. J Public Relat Res 2001; 13:163-85.

77. Sun A, Zhang J, Tsoh J, Wong-Kim E, Chow E. The effectiveness in utilizing Chinese media to promote breast health among Chinese women. J Health Commun 2007;12:157-71.

78. Lee H, Kim J, Han HR. Do cultural factors predict mammography behaviour among Korean immigrants in the USA? J Adv Nurs 2009;65:2574-84.

79. Wray RJ, McClure S, Vijaykumar S, Smith C, Ivy A, Jupka K, et al. Changing the conversation about prostate cancer among African Americans: results of formative research. Ethn Health 2009;14: 27-43.

80. Dutta MJ. Theoretical approaches to entertainment education campaigns: a subaltern critique. Health Commun 2006;20:221-31. 